\title{
Infection control since HIV
}

\section{David Croser}

BDA Indemnity Dental Advisor, describes how time has normalised the way we care for patients with a once frightening disease as another one hits the headlines

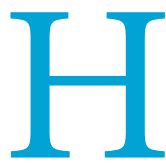
IV is the virus that can lead to acquired immunodeficiency syndrome or AIDS if not treated. There is no cure as yet but with proper medical care, HIV can be controlled. HIV is treated with antiretroviral therapy or ART. If taken as prescribed, ART can reduce the viral load so that it becomes undetectable. If it stays undetectable, patients can live long, healthy lives and have effectively no risk of transmitting HIV to an HIV-negative partner through sex, and more relevantly whilst being treated by a dental healthcare professional.

Today, someone diagnosed with HIV can live nearly as long as someone who does not have HIV. This is significant because there is an increasing number of HIV positive patients in the community whose bloodborne disease is controlled with ART- many of whom will also need dental treatment.

As a dentist, you have an obligation to provide dental care to those in need, including those who are or might be infected, and to offer the same high standard of care for all patients. It is unethical to refuse dental care to patients who disclose a positive diagnosis for a blood-borne virus (BBV) because you feel there could be an increased level of personal risk.

It is also illogical to think you are safer by refusing to treat patients who disclose they have a BBV; just as many undiagnosed carriers of BBVs attend for dental treatment and, because they have not received any treatment, they may actually present a higher risk of infection.

People living with HIV and the hepatitis viruses who are otherwise well, may be treated in general practice without the need for any restrictions or modification to their dental treatment.

Letting your patients know that you follow national guidelines on infection control - for example, by having a statement to this effect on your website or in your practice information leaflet or displaying a notice in the practice and encouraging them to ask questions will reassure them that you are confident about your infection control procedures.

Patients trust the dental team to use effective infection control measures to keep them safe. You can enhance the patient's trust by occasionally pointing out the precautions used in the surgery - e.g. the use of disposable items, sealed single use items like anaesthetic cartridges and newly sterilised handpieces). It is a good use of your time and tells the patient that you care about them and reinforces their trust in you, at a time when a new strain of corona virus has emerged.

The decontamination protocols required within the dental setting are defined in HTM 01-05 (England and Northern Ireland). ${ }^{1}$ It is not the intention of this article to describe those universal precautions. Suffice it to say that, maintaining a constant high standard is dependent on a dental team that understands the importance of this aspect of patient safety; routinely applying the latest protocol and checking their compliance by using a regular audit.

Following the identification of the retrovirus causing HIV, the dental profession and patients have been exposed to information about blood-borne diseases and the benefits of testing. The Internet and social media have helped to normalise the fact that some people do have a BBV; the fearful emotional reactions to HIV witnessed in the 1980s in the UK have now been replaced by an educated response based on reason.

The pendulum has now swung the other way so that any healthcare provider who exposes their patients to the risk of acquiring a BBV through a breach in the HTM 01-05 protocol, is likely to be shunned by society and exposed by the media.

This is exactly what happened in Leicester in 2014 when dentist Desmond D'Mello was suspended by the GDC over serious hygiene concerns and 22,000 patients from the practice were recalled and offered screening for HIV, HBV and HCV.

Since then, other cases have occasionally hit the headlines which inevitably challenges the trust placed in the dental profession. Patients may not understand what good infection control should look like, so take a moment to make them aware of what you do to keep them safe from catching an unwanted disease in your surgery.

\section{New diseases}

COVID-19 is currently having the same impact that AIDS generated when it first emerged, even though we are told that, in most cases, the effect of the disease will be mild. The fear of rapid spread and the absence of a cure makes it all the more important that healthcare workers set an example and respond to the challenge of limiting the spread of the disease by engaging with the science rather than emotions.

The standard use of infection control protocols is now augmented by the need to consider delaying treatment for those patients who are identified as being at risk of having been in contact with COVID-19.

Proposing a period of quarantine for a patient may be an unfamiliar step, but by following the DoH guidance ${ }^{2}$ primary care providers can protect themselves, their staff and the population at large.

The guidance will evolve as more is learnt about COVID-19 and the prospect of a vaccine becomes a reality. Indeed, if required, dental surgeries could provide a convenient setting from which to support a programme of mass vaccination. *

\section{Reference}

1. Health Technical Memorandum 01-05: Decontamination in primary care dental practices. Available online at: https://assets.publishing.service. gov.uk/government/uploads/system/uploads/ attachment_data/file/170689/HTM_01-05_2013.pdf (Accessed March 2020).

2. Public Health England. COVID-19: interim guidance for primary care. Available online at: https://www. gov.uk/government/publications/wn-cov-guidancefor-primary-care/wn-cov-interim-guidance-forprimary-care (Accessed March 2020).

\section{Advice}

BDA advice to dentists regarding COVID-19 is available at: www.bda.org/ news-centre/latest-news-articles/Pages/ Wuhan-novel-coronavirus-advice-fordentists.aspx

https://doi.org/10.1038/s41404-020-0359-y 